\title{
Revista \\ A prevenção do câncer e a promoção da saúde: um desafio para o Século XXI
}

\author{
Cancer prevention and health promotion: a challenge for the $21^{\text {st }}$ Century
}

La prevención de cancer y la promoción de salud: un desafio para el Siglo XXI

\section{Maria Elisa Wotzasek Cestari}

Enfermeira. Professora do Departamento de Enfermagem da Universidade Estadual de Londrina, Mestranda do Programa de

Enfermagem Fundamental da Escola de Enfermagem de Ribeirão Preto - USP

\section{Márcia Maria Fontão Zago}

Enfermeira. Professora Doutora da Escola de Enfermagem de Ribeirão Preto - USP

\section{RESUMO}

Este estudo é um ensaio sobre a prevenção do câncer, a promoção da saúde, e as estratégias de ações neste âmbito. Os avanços nas últimas décadas foram evidentes, mas a eqüidade em relação à promoção da saúde $e$ prevenção do câncer ainda não foi alcançada, sendo este o principal desafio para o século XXI. Nesse sentido, acredita-se que a valorização dos conhecimentos, das crenças, dos valores e das normas dos indivíduos, entendidas de uma forma ampla e heterogênea, é fundamental na reorientação dos serviços de saúde. Descritores: Promoção da saúde; Prevenção primária; Câncer/Neoplasias.

\section{ABSTRACT}

This study is an essay about cancer prevention, health promotion, and the action strategies in this field. The advances in the last decades were obvious, but the equitity in relation to health promotion and cancer prevention is not accomplished yet, being this the main challenge for the XXI century. In this meaning, it is believed that the valorization of knowledge, believes, values and norms of the individuals, understood in a large and similar way, are essential to the health services reorientation.

Descriptors: Health promotion, Primary prevention, Neoplasms.

\section{RESUMEN}

Este estudio es assaigment del na sobre la prevención de cáncer, promoción de la salud, y las estrategias de acción en este campo. Los adelantos en los últimos dacades eran obvios, pero el equitito respecto a la promoción de salud y la prevención del cáncer no es todavía cumplida y es este el desafío principal durante el XXI siglo. Este mening cree que la valorización de conocimiento, célibes, valores y normas de los individuos, entendida de una manera grande y similar, es assential a la salud repara reorientación.

Descriptores: Promoción de la salud; Prevención primaria; Neoplasias.

Cestari MEW, Zago MMF. A prevenção do câncer e a promoção de saúde: um desafio para o Século XXI. Rev Bras Enferm 2005 mar-abr; 58(2):218-21.

\section{INTRODUÇÃO}

O modelo de atenção à saúde que temos hoje ainda está centrado na assistência curativa individual, com foco no atendimento hospitalar. Este modelo não tem resolvido os problemas de saúde da nossa população, principalmente quando falamos do câncer, que tem apresentado um aumento em seus indicadores. Portanto, este estudo é um ensaio sobre a temática da prevenção do câncer e a da promoção da saúde, com foco nas estratégias de ações neste âmbito, onde se busca uma análise exploratória e crítica no sentido de propor-se um novo olhar para esta realidade.

A atenção primária, com suas ações de promoção, prevenção e detecção precoce do câncer, deve ser vista como prioritária à atenção terciária( ${ }^{(1,2)}$. A Organização Mundial da Saúde ${ }^{(3)}$ prioriza as ações de prevenção no cuidado com as condições crônicas, enfatizando que é possível prevenir a maioria destas, e que, assim, toda a interação de saúde deve incluir a prevenção. Entretanto, nos deparamos com a dificuldade de adesão dos indivíduos aos comportamentos preventivos preconizados pelo modelo biomédico, principalmente aqueles com condições socioeconômicas mais baixas.

Entende-se por comportamentos preventivos em saúde (CPS) aqueles comportamentos empreendidos pelos indivíduos para aumentar ou manter a própria saúde. Há instâncias inumeráveis de CPSs: a limpeza diária dos dentes, uso de cintos de segurança, redução da gordura e açúcar na dieta, ou prática de atividade física são alguns exemplos( ${ }^{(4)}$.

O comportamento preventivo está ligado a fatores sociais, psicológicos, ambientais ${ }^{(5)}$ e ainda, 
culturais ${ }^{(6)}$. Sendo que os CPSs provavelmente podem variar positiva ou negativamente, dependendo da influência destes fatores no estilo de vida dos indivíduos.

Em algumas culturas, como a nossa, a palavra câncer é tabu e se a morte for conseqüência de um câncer, muitas vezes a causa atribuída será outra. As superstições são numerosas, até mesmo nas nações desenvolvidas onde o nível educacional para a saúde é considerado satisfatório ${ }^{(7)}$. No Brasil, pode-se exemplificar por um lado, a dificuldade de algumas mulheres em submeterem-se ao exame ginecológico por vergonha de expor sua genitália, ou por outro lado, o hábito saudável e de proteção ao câncer, que é a ingestão de peixes e soja pelos indivíduos orientais.

Em estudos da área da antropologia médica o CPS é considerado um sistema cultural e de significados que possui uma organização interna dos saberes individuais, resultando num modo próprio do indivíduo explicar e buscar a resolução de seus problemas de saúde, como por exemplo, por meio de determinados comportamentos ${ }^{(8)}$.

Mais recentemente, encontra-se o conceito de atitude positiva em prevenção que é definindo como uma procura do indivíduo por cuidado, independentemente dele ou do grupo a quem ele pertence, estar vivenciando um problema de saúde. Esta atitude é determinada pelas crenças e percepções do indivíduo sobre o que é saúde, doença, prevenção, e também pelas experiências vivenciadas por ele, seja para prevenção, manutenção ou tratamento de sua saúde. O conceito é composto por duas dimensões que podem influenciar as atitudes. A primeira refere-se às condições concretas de existência do indivíduo, caracterizada pela condição sócio-econômica. A segunda contempla as percepções e comportamentos em saúde, caracterizadas pela percepção do problema de saúde e procura por cuidado de saúde pelo indivíduo ${ }^{(9)}$

\section{A PREVENÇÃO E A PROMOÇÃO DA SAÚDE}

Outros conceitos que permeiam esta temática, sendo importante a definição dos mesmos são a prevenção e promoção da saúde. A palavra prevenção tem origem no Latim praeventione: vir antes, tomar a dianteira; traduz-se pelo ato de prevenir-se, premeditar, dispor-se previamente ou ter opinião antecipada ${ }^{(10,11)}$. A prevenção, na área da saúde, é composta por ações de caráter primário e genérico, tais como a melhoria das condições de vida, redução da suscetibilidade das pessoas às doenças e educação sanitária. A prevenção se dá também através da detecção precoce das doenças, do seu tratamento adequado e nas ações destinadas a minimizar as suas conseqüências ${ }^{(12)}$. O termo prevenção tem sido intensamente utilizado no âmbito da saúde, entretanto, na prática tem sido tão pouco efetivado ${ }^{(12)}$. Nesse contexto é inerente a dificuldade das relações com modelos e padrões de reconhecimento e valorização dos aspectos culturais. Visto que, qualquer ação de prevenção deveria estar atenta aos valores, atitudes e crenças dos grupos sociais a quem a ação se dirige, ou seja, aos seus aspectos culturais $^{(13)}$

Paralelo a esta discussão, o conceito de promoção da saúde (PS) surgiu no Canadá em 1974, motivado pelas limitações da economia, visto o custo elevado dos tratamentos curativos das doenças e pelas limitações da atenção médica à população ${ }^{(14)}$. 0 início do seu fortalecimento acontece a partir da I Conferência Internacional sobre PS, no ano de 1986 em Ottawa. Na carta produzida nesta conferência a PS foi entendida como o processo de capacitação da comunidade para atuar na melhoria da sua qualidade de vida e saúde, incluindo uma maior participação no controle desse processo.

Nesse momento a eqüidade foi considerada o principal foco a ser atingido para se ter acesso à PS, entendendo-se que conseguir a eqüidade consiste em eliminar as diferenças desnecessárias, evitáveis e injustas que limitam as oportunidades do indivíduo de atingir o direito do bem estar ${ }^{(15)}$.

Em 1988, na Austrália, uma nova conferência priorizou, pela Declaração de Adelaide, as políticas públicas saudáveis, ou seja, apoio à saúde da mulher, alimentação e nutrição, políticas de redução do consumo do tabaco e do álcool e criação de ambientes favoráveis ${ }^{(15)}$.

Em 1991, na Suécia, a III conferência fortalece ainda mais a temática, com ênfase na criação de ambientes favoráveis à saúde, mantendo a busca pela eqüidade e educação, além de propor o aumento do poder de decisão dos indivíduos e da participação comunitária como estratégia para ter-se um processo democrático de PS.

Em 1992, outra conferência realizada em Bogotá, na Colômbia destaca os problemas enfrentados pela América Latina, como a necessidade de solidariedade e eqüidade social como condições indispensáveis para a saúde e o desenvolvimento ${ }^{(15)}$.

Em 1997, a Declaração de Jacarta, produto da IV Conferência Internacional sobre PS destaca que esta é um processo que permite às pessoas maior controle sobre sua saúde e, assim, tenham maior poder de melhorá-la. Sua meta primordial foi aumentar as expectativas de saúde e reduzir suas diferenças entre países e grupos. A pobreza foi tida como a maior ameaça à saúde. Portanto estabeleceu-se cinco prioridades para a PS no século XXI: promover a responsabilidade social para com a saúde; aumentar os investimentos para fomentar a saúde; consolidar e expandir parcerias em prol da saúde; aumentar a capacidade comunitária e dar direito de voz ao indivíduo e; conseguir uma infra-estrutura para a PS ${ }^{(15)}$.

E finalmente, a V Conferência aconteceu no México em 2000, tendo como principais objetivos demonstrar a importância da PS principalmente para indivíduos que vivem em condições adversas; incluir a saúde nas agências de desenvolvimento; estimular alianças entre os diferentes atores em todos os níveis sociais. Os principais resultados desta conferência foram a reafirmação da importância da PS e da busca da eqüidade e ainda a importância das mulheres no desenvolvimento de ações de $\mathrm{PS}^{(16)}$.

Em síntese a conferências criaram metas que provavelmente poderão ajudar os países a diminuir as injustiças e desigualdades, mas também, têm contribuído por meio dos avanços ideológicos, políticos e conceituais, para a evolução das propostas para melhoria a qualidade de vida dos indivíduos. E para que se alcance a qualidade de vida é necessário atuar-se sobre as condições sociais que são determinantes da saúde ${ }^{(17)}$.

Nesse sentido é possível diferenciar-se os conceitos de prevenção e PS, considerando que a prevenção é definida por ações direcionadas a evitar o surgimento de doenças específicas, através do controle de transmissão e redução do risco de doenças degenerativas ou outros agravos específicos. Em contrapartida, a promoção tem um conceito mais amplo, que enfatiza a transformação das condições de vida e de trabalho, por mudanças profundas na forma de articular e utilizar 0 conhecimento na construção e operacionalização das práticas de saúde, visando aumentar a saúde e o bem-estar gerais, não relacionados com alguma doença específica ${ }^{(18)}$. Ou seja, fazer a PS é permitir-se a um permanente e contínuo processo de reflexão sobre a articulação e coerência, entre a produção discursiva e a práxis, por meio de uma postura crítica e positiva para viver e mudar posturas e ações ${ }^{(17)}$.

\section{A PROMOÇÃO DA SAÚDE E A PREVENÇÃO DO CÂNCER COMO UM DESAFIO ATUAL}

A produção do conhecimento e as mudanças da práxis da saúde têm sido mais efetivas nos últimos anos. Observa-se a apropriação pelos indivíduos dos saberes sobre saúde, riscos e doenças, difundidos pelos meios de comunicação de massa. É um fato a revolução nas comunicações, a expansão, praticamente ao infinito, pela Internet e a 
circulação de informações sobre saúde, risco, danos, exames, terapias e práticas visando a preservação e recuperação da saúde no plano individual, tornando o próprio sujeito responsável por decisões e ações que afetem direta ou indiretamente sua saúde ${ }^{(6)}$.

Em países desenvolvidos esta já é uma realidade palpável. Na Inglaterra as pessoas estão sendo encorajadas, nas consultas médicas, a responsabilizar-se pela própria saúde, por meio da adoção de comportamentos saudáveis, sendo esta uma característica atual implícita no cuidado preventivo na atenção à saúde primária ${ }^{(19)}$

No Canadá as ações de saúde também deslocam a ênfase da prevenção de doenças para a PS e esta ganha uma importância capital, sendo definidora de um novo modelo que incorpora políticas públicas saudáveis e mudanças em estilos de vida(6). Esta é, portanto, uma tendência mundial, onde se destaca a crescente importância das atitudes individuais para a promoção, prevenção e proteção de doenças, além da disseminação paralela dos fatores de risco à saúde associando sua prática, ao crescente peso da razão econômica ${ }^{(14)}$.

Entretanto, a mercantilização da saúde, com a comercialização em larga escala de informações e produtos dirigidos à preservação da vida saudável, do viver com saúde, na perspectiva do consumo individualizado de produtos e serviços, não significará necessariamente uma racionalização da vida no sentido da incorporação exclusiva de um saber científico, instrumental. Pelo contrário, a julgar pelas características e multiplicidade de informações, o cuidado à saúde no plano individual não deixará de manter o apelo ao simbólico, ao mágico, ao sobrenatural, ao místico, e mesmo ao metafísico(6).

As condições de vida globalizadas, hoje, em hipótese, são configuradas como forma de sofrimento social que pode ser traduzida como uma pandemia de saúde mental, resultante de mudanças na política, economia e cultura. A propagação do tabaco e do álcool pela mídia e os problemas de saúde associados ao seu uso são um exemplo ${ }^{(8)}$.

É provável que uma das principais características das práticas de saúde no futuro será a ênfase concedida à pesquisa e principalmente, às ações em prevenção. Assim, uma exploração das possibilidades colocadas para o século XXI pressupõe análise das tendências da produção de conhecimentos e do desenvolvimento de práticas de prevenção, promoção e vigilância da saúde, levando em conta a identificação das perspectivas econômicas, políticas e sociais que se apresentam no momento em que se consolida o processo de globalização da economia e da cultura ${ }^{(6)}$.

Assim, o câncer é indubitavelmente um problema de saúde pública, incluído entre as primeiras causas de morte nas diferentes regiões do nosso país. Hoje este agravo é a segunda causa de morte por doença no Brasil, perdendo somente para as doenças do coração, e seguido das doenças cerebrovasculares ${ }^{(20)}$. Estima-se que em 2003, no Brasil, ocorreram 402.190 casos novos e 126.960 óbitos por câncer. Esperase encontrar para o sexo masculino 186.155 casos novos e 68.350 óbitos, enquanto que, para o sexo feminino, 216.035 casos novos e 58.610 óbitos ${ }^{(20)}$.

As maiores taxas de incidência entre os homens foram provavelmente devidas ao câncer de pele não melanoma, próstata, pulmão, estômago e cólon e reto enquanto que nas mulheres, destacam-se as neoplasias malignas da pele não melanoma, mama, colo do útero, cólon, reto e estômago. Provavelmente o câncer de pulmão foi a primeira causa de morte por câncer no sexo masculino, seguido do câncer de próstata, estômago, esôfago, cólon e reto. Estima-se que o câncer da mama feminina manter-se-á como a primeira causa de morte em mulheres, seguido pelo câncer de pulmão, cólon, reto, colo do útero e estômago ${ }^{(20)}$.

Analisando-se o panorama apresentado, a importância do câncer como um problema de saúde pública em nosso país torna-se evidente, desencadeando uma discussão sobre a prevenção do câncer, 0 comportamento preventivo a este agravo e a PS.

Conceitualmente, prevenir o câncer consiste em reduzir ao mínimo ou eliminar a exposição aos agentes carcinogênicos além de minimizar a suscetibilidade individual aos efeitos destes agentes ${ }^{(21)}$. Este é um conceito simplificado. Poderiam ser acrescentados a ele os aspectos sociais, econômicos e culturais.

Para prevenir o câncer a população deve ser informada sobre os comportamentos de risco, os sinais de alerta e a freqüência da prevenção. Mas, além disto, é importante a capacitação dos recursos humanos que atuam nesta área, buscando uma reorientação para a cultura do câncer e consequentemente mudanças na práxis destes profissionais ${ }^{(22)}$.

Em oncologia encontra-se o termo prevenção classificado em níveis primário e secundário. A prevenção primária situa-se no período anterior à doença, incluindo medidas inespecíficas de proteção de indivíduos contra riscos e danos ${ }^{(6)}$. Refere-se a toda e qualquer ação voltada para redução da exposição da população a fatores de risco da doença, tendo como objetivo reduzir a sua ocorrência, por meio da promoção da saúde e proteção específica ${ }^{(23)}$.

Portanto, a prevenção primária divide-se nas ações de promoção e nas ações de proteção específicas contra fatores de riscos para o câncer, sendo que a promoção da saúde se relaciona às medidas inespecíficas da prevenção primária, como luta contra o tabagismo, orientações sobre dieta saudável e proteção solar e a proteção específica, refere-se às ações mais diretas, como a vacinação e o exame de Papanicolaou.

O Instituto Nacional do Câncer ${ }^{(23)}$ considera como principais fatores de risco para o câncer: o tabagismo; 0 alcoolismo; os hábitos alimentares, principalmente em relação ao consumo de alimentos ricos em gordura, nitritos, alcatrão e aflatoxina; as radiações, sendo estas as ionizantes e as radiações ultravioletas natural, provenientes do sol; 0 uso de medicamentos, que podem ter efeito carcinogênico ou ainda supressores imunológicos; 0 uso de hormônios e fatores reprodutivos; o contato com os agentes infecciosos e parasitários; a exposição ocupacional, com exposição a agentes químicos, físicos ou biológicos e; a poluição do ambiente geral.

A prevenção primária destaca-se como a melhor alternativa quando comparada ao diagnóstico ou mesmo ao tratamento do câncer. Visto que apesar de sermos incapazes de mudar nossa predisposição genética, podemos ter a possibilidade de intervenção para prevenir exposições e os fatores causais do câncer ${ }^{(24)}$.

A prevenção secundária é o rastreamento (screening) do câncer. Entende-se por rastreamento uma avaliação de indivíduos assintomáticos, para classificá-los como candidatos a exames mais refinados de avaliação, com o objetivo de descobrir um câncer oculto ou uma afecção pré-maligna que pode ser curada com tratamento. (BRASIL, 2002).

O rastreamento é a única estratégia potencialmente capaz de reduzir a mortalidade em dois grupos de câncer: aqueles encontrados com freqüência, para os quais o tratamento, se metastizados, não é curativo e aqueles cujas causas não são conhecidas e, portanto, a possibilidade de prevenção primária não existe. Ele está baseado na suposição de que o diagnóstico precoce do câncer resultará na sua descoberta antes que ocorram metástases fatais. O rastreamento é factível para diversos tipos de câncer incluindo o de mama, o da cérvice uterina, o de intestino grosso, o de estômago e o melanoma maligno(25). São exemplos de ações para detecção precoce a colpocitologia, a momografia e 0 autoexame da boca.

É indiscutível que a prevenção do câncer é uma prática possível. As práticas de prevenção, entretanto, não estão sendo aplicadas em sua plenitude. Estas dependem da vontade dos políticos, da sensibilização dos profissionais de saúde, e da motivação dos pacientes ${ }^{(26)}$. Ainda hoje, 
muitas mulheres continuam morrendo por câncer de colo uterino por falta de detecção e diagnóstico precoce, ou seja, as medidas adotadas até 0 momento, não tiveram o impacto desejável(27).

Sendo assim, quatro programas de prevenção e detecção precoce deveriam ser considerados prioritários à nossa realidade. A prevenção dos cânceres do colo uterino, de mama, de boca e de pele. Porém, estes programas continuam a enfrentar problemas para se desenvolver.

\section{CONSIDERAÇÕES FINAIS}

Enfim, o ensaio apresentado representa 0 que se tem feito para efetivar-se as ações de prevenção do câncer e promoção da saúde. Seria equânime considerar-se que houve avanços nas últimas décadas,

\section{REFERÊNCIAS}

1. Starfield B. Atenção primária: equilíbrio entre necessidades de saúde, serviços e tecnologia. Brasília (DF): UNESCO; 2002.

2. Paim JS. Vigilância da saúde: tendências de reorientação de modelos assistênciais para a promoção da saúde. In: Czeresnia D, Freitas $\mathrm{CM}$, organizadores. Promoção da saúde: conceitos, reflexões, tendências. Rio de Janeiro (RJ): Fiocruz; 2003. p. 161-74.

3. Organização Mundial da Saúde. Cuidados inovadores para condições crônicas: componentes estruturais de ação - relatório mundial Brasília (DF): OMS; 2003.

4. Pitts M. The psychology of preventive health. London(UK): Routledge; 1996

5. Langlie JK. Social networks, health beliefs, and preventive health behavior. J Health Social Behavior 1977 sep;18: 244-60.

6. Teixeira C. O futuro da prevenção. Salvador (BA): Casa da Qualidade; 2001

7. Sherman Júnior C D. Os aspectos psicossociais do câncer. In: Love RR, editor. Manual de oncologia clínica. $6^{a}$ ed. São Paulo (SP): Fundação Oncocentro de São Paulo; 1999. p. 598-606.

8. Kleinman A. Experience and its moral modes: culture, human conditions, and disorder. San Francisco (USA): Stanford University; 1998.

9. Maciel AA. A procura por cuidado de saúde: o papel das crenças e percepções de mulheres na vivência do processo saúde-doença [tese]. São Paulo (SP): Faculdade de Saúde Pública, Universidade de São Paulo; 1999

10. BuenoS. Minidicionário da língua portuguesa. São Paulo(SP): FTD; 2000

11. Ferreira $A B H$. Novo Aurélio século $X X I$ : o dicionário da língua portuguesa. $3^{a}$ ed. Rio de Janeiro (RJ): Nova Fronteira; 1999.

12. Silveira ML. Família, cultura e prevenção. In: Anais do Seminário sobre Cultura, Saúde e Doença; 2000; Londrina (PR), Brasil. Londrina (PR): [s.n]; 2003. p 171-82.

13. Minayo MCS. O desafio do conhecimento: pesquisa qualitativa em saúde. $2^{a}$ ed. São Paulo (SP): Hucitec; 1993

14. Freitas CM. A vigilância da saúde para a promoção da saúde. In: Czeresnia D, Freitas CM, organizadores. Promoção da saúde: conceitos, reflexões, tendências. Rio de Janeiro (RJ): Fiocruz; 2003. p.141-59

15. Ministério da Saúde (BR). Fundação Oswaldo Cruz. Promoção da saúde: carta de Ottawa, declaração de Adelaide, declaração de Sundsvall e declaração de Bogotá. Brasília (DF): Ministério da Saúde; 1996.

16. Ministério da Saúde (BR). Projeto Promoção da Saúde. Promoção da saúde: carta de Ottawa, declaração de Adelaide, declaração de mas a qualidade de vida e a vida saudável tão sonhadas pelos indivíduos e preconizadas pelo modelo biomédico ainda não foram conquistadas com eqüidade, sendo este talvez o principal desafio para o século XXI. Nesse sentido, acredita-se que a valorização dos conhecimentos, das crenças, dos valores e das normas dos indivíduos, entendidas de uma forma ampla e heterogênea ${ }^{(28)}$, são fundamentais na reorientação dos serviços de saúde. Propõe-se então a realização de novas pesquisas na área da promoção da saúde e prevenção do câncer, bem como, mudanças na educação formal da população e no ensino específico dos profissionais da área de saúde, uma vez que, estes podem proporcionar uma maior adesão aos programas de promoção da saúde e prevenção do câncer pelos indivíduos.

Sundsvall e declaração de Santafé de Bogotá, declaração de Jacarta rede de megapaíses e declaração do México. Brasília (DF): Ministério da Saúde; 2001.

17. Guimarães MA. et al. Promoção da saúde: as conferências internacionais de promoção da saúde. [citado em 7 jun 2004]. Disponível em: URL: http://www.sobral.ce.gov.br/ saudedafamilia/ Publicacoes/PromoSaude/conferencias_internacionais.htm

18. Czeresnia $D$. O conceito de saúde e a diferença entre prevenção $e$ promoção. In: Czeresnia D, Freitas CM, organizadores. Promoção da saúde: conceitos, reflexões, tendências. Rio de Janeiro (RJ): Fiocruz; 2003. p.39-53.

19. Conner M, Norman P. Predicting health behaviour. Philadelphia (PA): Open University Press; 1998.

20. Ministério da Saúde (BR). Instituto Nacional do Câncer. Estimativas da incidência e mortalidade por câncer no Brasil. [citado em 21 mar 2004]. Disponível em: URL: http://www.inca.gov.br/estimativas/2003/index

21. Organização Mundial de La Salud. Programas nacionales de lucha contra o câncer - directrices sobre política y gestion. Genebra (SWT): OMS; 1995.

22. Vanzin AS, Nery MES. Câncer: problema de saúde pública e saúde ocupacional - atuação do enfermeiro na prevenção do câncer. Porto Alegre (RS): RM\&L; 1997.

23. Ministério da Saúde (BR). Instituto Nacional do Câncer. Ações de enfermagem para o controle do câncer: uma proposta de integração ensino-serviço. $2^{\mathrm{a}}$ ed. Rio de Janeiro (RJ): INCA; 2002.

24. Neugut Al. Prevenção primária. In: Love RR, editor. Manual de oncologia clínica. $6^{a}$ ed. São Paulo (SP): Fundação Oncocentro; 1999. p.93-102.

25. Gill PS, Tattersall, MHN. Rastreamento e detecção precoce. In: Love RR, Editor. Manual de oncologia clínica. $6^{a}$ ed. São Paulo (SP): Fundação Oncocentro; 1999. p.117-38.

26. Coelho FR.G. A prevenção do câncer. Acta Oncol Bras 1994 jun; 149(3):105-18.

27. Zeferino L, Galvão L. Prevenção e controle do câncer de colo uterino: por que não acontece no Brasil? In: Galvão L, Diaz J, organizadores. Saúde sexual e reprodutiva no Brasil. São Paulo (SP): Hucitec; 1999. p. 346-65.

28. Langdon EJ. Cultura e processos de saúde e doença. In: Seminário sobre Cultura, Saúde e Doença; 2000; Londrina (PR), Brasil. Londrina (PR): [s.n.]; 2003. p 91-107. 